\title{
Evaluation of gridded scanning ARM cloud radar reflectivity observations and vertical doppler velocity retrievals
}

\author{
K. Lamer, A. Tatarevic, I. Jo, and P. Kollias \\ Department of Atmospheric and Oceanic Sciences, McGill University, Montreal, Canada \\ Correspondence to: K. Lamer (katia.lamer@mail.mcgill.ca) \\ Received: 20 August 2013 - Published in Atmos. Meas. Tech. Discuss.: 8 November 2013 \\ Revised: 5 March 2014 - Accepted: 10 March 2014 - Published: 29 April 2014
}

\begin{abstract}
The scanning Atmospheric Radiation Measurement (ARM) cloud radars (SACRs) provide continuous atmospheric observations aspiring to capture the 3-D cloudscale structure. Sampling clouds in 3-D is challenging due to their temporal-spatial scales, the need to sample the sky at high elevations and cloud radar limitations. Thus, a suggested scan strategy is to repetitively slice the atmosphere from horizon to horizon as clouds advect over the radar (Cross-Wind Range-Height Indicator - CW-RHI). Here, the processing and gridding of the SACR CW-RHI scans are presented. First, the SACR sample observations from the ARM Southern Great Plains and Cape Cod sites are post-processed (detection mask, gaseous attenuation correction, insect filtering and velocity de-aliasing). The resulting radial Doppler moment fields are then mapped to Cartesian coordinates with time as one of the dimensions. Next the Cartesian-gridded Doppler velocity fields are decomposed into the horizontal wind velocity contribution and the vertical Doppler velocity component. For validation purposes, all gridded and retrieved fields are compared to collocated zenith-pointing ARM cloud radar measurements. We consider that the SACR sensitivity loss with range, the cloud type observed and the research purpose should be considered in determining the gridded domain size. Our results also demonstrate that the gridded SACR observations resolve the main features of low and high stratiform clouds. It is established that the CWRHI observations complemented with processing techniques could lead to robust 3-D cloud dynamical representations up to 25-30 degrees off zenith. The proposed gridded products are expected to advance our understanding of 3-D cloud morphology, dynamics and anisotropy and lead to more realistic 3 -D radiative transfer calculations.
\end{abstract}

\section{Introduction}

Ground-based and space-borne cloud radars and lidars are the primary instruments used to resolve clouds' vertical structure as well as their dynamical and microphysical properties. Through ongoing research and development over the last two decades, millimeter-wavelength radar sophistication and availability in different atmospheric research platforms has significantly increased (Kollias et al., 2007). During this period, the bulk of the cloud radar observations were collected in a zenith-pointing mode (profiling). It is true that scientists and modelers have cleverly used vertical-column radar information complemented with lidar and radiometer information to improve our knowledge of clouds (Shupe et al., 2011; Rémillard et al., 2012) as well as their parameterization in cloud models of variable scales ranging from large eddy simulations to global climate models (Randall et al., 1996; Somerville and Iacobellis, 1999; Qian et al., 2012). However, the lack of actual 3-D cloud structure information hinders our ability to quantify the 3-D radiative effects and measure the cloud field anisotropy, and introduces uncertainties in retrievals of cloud properties (Hinkelman et al., 2005; Davis et al., 1997; Zinner and Mayer, 2006).

Continual advancements in millimeter-wavelength radar technology permitted the deployment of a number of scanning cloud radars (Mather and Voyles, 2013) that can operate off zenith, thus providing a hemispheric view of the cloudy atmosphere. Scanning radars are not new and have been widely used by the weather community in forecasting and research applications. However, weather radars and cloud radars have different coverage objectives (mapping precipitation over large area vs. providing high-resolution measurements over the site), antenna scan rates (much lower 
for cloud radars due to their narrow beam width) and targets with different temporal-spatial characteristics (Kollias et al., 2013a). Weather radars mostly use volume coverage patterns designed to provide large horizontal coverage at low levels using a sequence of constant elevation PPI (plane-position indicator) scans. By contrast, scanning cloud radars need to sample the part of the atmosphere near and above the groundbased facility, and thus execute innovative scanning strategies such as the cross-wind range-height indicator scan strategy (CW-RHI). This scanning strategy relies on a sequence of constant azimuth RHI scans with changing elevation (0$180^{\circ}$ ) to slice the cloudy atmosphere as it advects over the radar (CW-RHI, Kollias et al., 2013a). Using a sequence of these scans, with time as one of the dimensions, we can interpolate the 3-D morphological (reflectivity) and dynamical (Doppler velocity) cloud structure to a Cartesian grid. Gridded scanning cloud radar observations would provide complete information on cloud overlap conditions, as well as allow a new view of cloud life cycle analysis. They could also tighten constraints on radiative transfer calculations. In addition, cloud 3-D dynamical representation can be used to characterize cloud-scale turbulence including the vertical and horizontal organization of large eddies, and their extent, tilt and orientation with respect to the mean flow and large-scale meteorology.

Here, CW-RHI and collocated profiling cloud radar observations are used to evaluate (i) the reconstruction of the 3-D radar reflectivity field and (ii) the retrieval of the 3-D vertical Doppler velocity field after the removal of the horizontal wind contribution. It is established that the $\mathrm{CW}$-gridding does not alter the data and facilitates interpretation. It is also determined that the reduction of radar sensitivity with range should be one of the factors determining the grid space size. Moreover, the proposed decomposition technique to retrieve radar plane wind contribution resulted in a range of confident vertical velocity retrievals extending to 25-30 degrees.

The observational settings, the radar characteristics, scan strategy and raw data post-processing are described in Sect. 2. The Cartesian CW-gridding algorithm is described and evaluated in Sect. 3. Section 4 contains the description and evaluation of a technique to isolate vertical Doppler velocity from observed radial Doppler velocity. Summary and recommendations are provided in the last section.

\section{Observation and raw data post-processing}

The US Department of Energy created the Atmospheric Radiation Measurement (ARM) program in the mid 90s aiming, amongst other things, to increase our understanding of the effect of clouds on radiative fluxes and, accordingly, to improve our modeling capabilities (Stokes and Schwartz, 1994). Currently, the ARM program deploys multiple fixed and mobile facilities designed to observe various cloud types and connect cloud-scale processes and properties to the large-scale meteorology (Mather and Voyles, 2013). The large array of collocated instruments on site provides a unique opportunity to examine agreement in observational techniques among instruments, as well as to evaluate the accuracy of different methodologies applied to measurements.

This study's data set was obtained at two sites. The first is the ARM mobile facility in Cape Cod, MA; data was obtained during the Two-Column Aerosol Project (TCAP) field campaign, which took place for a period of 12 months starting in summer 2012 and aimed at quantifying aerosol properties, radiation and cloud characteristics. The case study presented here is from 19 November 2012. The close proximity of this site to the ocean enabled observations of marine stratus because low-level winds often advect these clouds shoreward. The second site is the Southern Great Plains (SGP), OK, an ARM fixed site, which is characterized by a relatively homogeneous geography and a wide variability of atmospheric conditions and cloud types. The case study presented here is from 7 December 2012.

The data of this study was obtained with the Ka-band $(35.3 \mathrm{GHz})$ Scanning ARM cloud radar (SACR), a fully coherent dual-frequency, dual-polarization Doppler radar mounted on a common scanning pedestal paired with either a W-band $(94.0 \mathrm{GHz})$ or an X-band $(9.7 \mathrm{GHz})$ radar, depending on the location of deployment (Fig. 1a) (only data from the Ka-band radar was used for this study). The specific settings of the instruments for each site are provided in Table 1. The radar operated the Cross-Wind Range-Height Indicator (CW-RHI) scan strategy, which consists of operating multiple horizon-to-horizon scans at an azimuth perpendicular to the leading wind directions (Cape $\operatorname{Cod} 225^{\circ}$ and SGP $180^{\circ}$ ) for a chosen time period (Fig. 1b). In the cases presented, 60 horizon-to-horizon scans were collected in $19 \mathrm{~min}$. An average of 20 sets of 19 min duration were collected per day.

Both sites were also equipped with profiling cloud radars: the W-band (95 GHz) ARM cloud radar (WACR) at Cape Cod and the Ka-band ARM zenith-pointing radar (KAZR) at the SGP. These zenith-pointing Doppler radars collected continuous data over the period of the field experiments. Although providing a very good temporal coverage, their view was limited to 2-D time-height information. More specific information about these instruments is also provided in Table 1 .

Finally sounding information was collected with a radiosonde four times per day, approximately at 05:30, 11:30, 17:30 and 23:30 UTC.

The first step in SACR data post-processing is the identification of radar volumes containing atmospheric returns (clouds, insects and ground clutter). At each radar profile, the receiver noise is identified and a preliminary binary feature mask is estimated using a power threshold. Using a rangeelevation spatial filter, a refined feature mask is estimated (Clothiaux et al., 1995; Hildebrand and Sekhon, 1974).

Second, the nearest (in time) atmospheric sounding is used to extract the profiles of water vapor, pressure and 

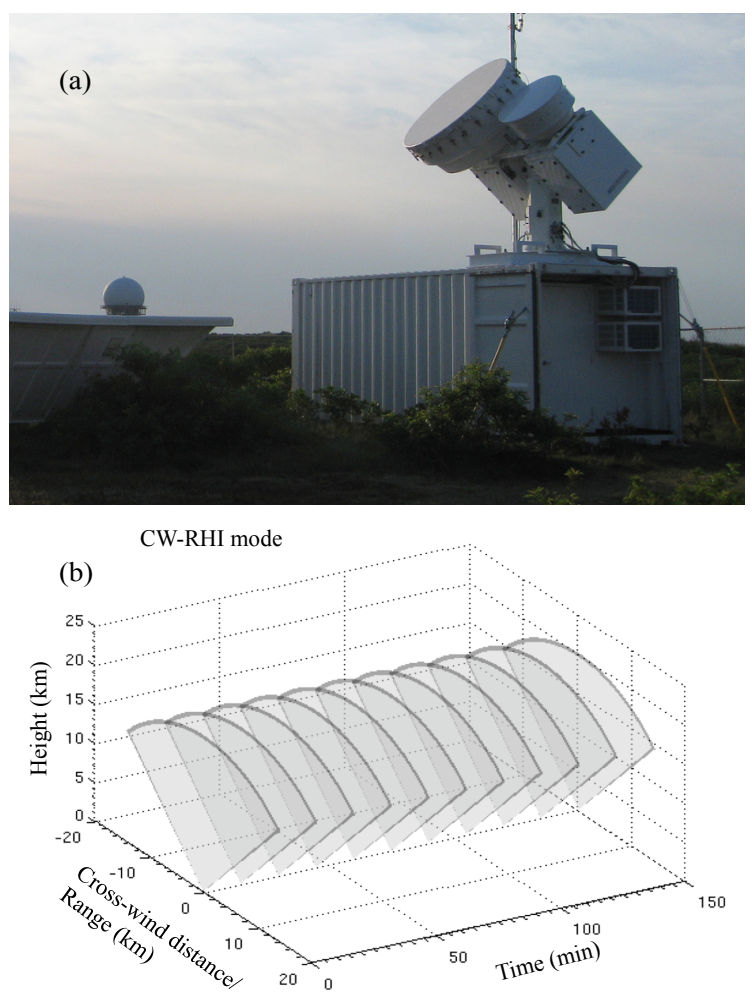

Fig. 1. (a) The Ka/W-SACR at Cape Cod during the ARM mobile facility deployment. A similar system is deployed at the ARM SGP site. (b) Schematic of the CW-RHI scan strategy.

temperature, which, along with standard atmospheric $\mathrm{O}_{2}$ and $\mathrm{N}_{2}$ concentrations, are projected onto the SACR slant line-of-sight. The projected profiles are used as input to the Rosenkranz (1998) line-by-line absorption model to estimate the two-way gaseous attenuation along all SACR beam paths used to correct reflectivity observations.

The next step in the Ka-SACR data post-processing is the application of an insect filter to remove non-meteorological returns in the boundary layer. The insect filter is applied to profiles where cloud bases are detected within a $1 \mathrm{~h}$ window and heights where the temperature is higher than $5^{\circ} \mathrm{C}$. Where these conditions are satisfied, the algorithm uses the linear depolarization ratio measurements to distinguish insect returns from clouds.

Finally, the SACR radial Doppler velocities are unfolded. The velocity convention adopted here is as follows: positive velocities are away from the instrument and negative velocities are towards the instrument. The nearest atmospheric soundings' wind velocity magnitude and direction serve as an estimate of the actual radial velocities on the radar plane. This estimate is then compared to the radar velocity observations and Nyquist velocity to make a first guess at the number of foldings which is then refined using a cost function that ensures smoothness across the range. A detailed methodology of the quality-control processing of the SACR observations
Table 1. Specification of radars used in this study: Ka-band ARM Zenith Radar (KAZR), W-band ARM cloud radar (WACR), Ka-band Scanning Cloud Radar (Ka-SACR). Transmitter types: traveling-wave tube amplifier (TWTA) and extended interaction klystron amplifier (EIKA).

\begin{tabular}{lrrr}
\hline Parameters & KAZR & WACR & Ka-SACR \\
\hline Transmitter type & TWTA & EIKA & EIKA \\
Peak power output $(\mathrm{W})$ & 150 & 1600 & 2200 \\
Duty cycle (\%) & 25.0 & 1.0 & 5.0 \\
Center frequency (GHz) & 34.86 & 95.00 & 35.29 \\
Antenna size (m) & 3.0 & 1.22 & 1.82 \\
3-dB beamwidth $\left({ }^{\circ}\right)$ & 0.19 & 0.18 & 0.33 \\
Range resolution (m) & 30 & 43 & 30 \\
Temporal resolution $(\mathrm{s})$ & 2 & 2 & 2 \\
Nyquist velocity (m s ${ }^{-1}$ ) & 5.95 & 8.0 & 10.53 \\
Integration time (s) & 2 & 2 & 0.037 \\
Sensitivity (dBZ)* & -50 & -50 & -50 \\
\hline * Accounting for signal integration time, at $1 \mathrm{~km}$. & &
\end{tabular}

(feature mask, gaseous attenuation correction, insect filter and Doppler velocity unfolding) can be found in Kollias et al. (2013b).

\section{CW-gridding algorithm}

The post-processed CW-RHI SACR radar observables (Sect. 2) are used as inputs to the $\mathrm{CW}$-gridding algorithm described here.

\subsection{Radar sensitivity}

A challenge closely related to the quality of gridded data is radar sensitivity. In weather radars, the key challenges in interpreting long-range observations are related to Earth's curvature and the broadening of the radar beam. The drop in radar sensitivity with the square of the distance from the radar is not a major concern since weather radars' operational mandate is related to the detection of intense echoes from severe weather. For the Ka-SACR, large sampling volumes and long ranges are not an issue since the radar has a very narrow beamwidth $\left(0.33^{\circ}\right)$ and typically operates within a range of $20 \mathrm{~km}$. Given that one of the main operational mandates of the Ka-SACR is to provide detailed characterization of hydrometeor locations in 3-D and since the radar-lidar instrument synergy for the determination of hydrometeor layers is not possible (Clothiaux et al., 2000), the determination of the cloud locations is based on Ka-SACR observations only. Thus, the drop of the radar sensitivity with the square of the distance from the radar can lead to significant range-dependent biases when it comes to fundamental cloud properties such as cloud fraction and cloud boundaries.

The impact of reduced sensitivity with range on cloud fraction is shown in Fig. 2. All totally overcast stratocumulus 


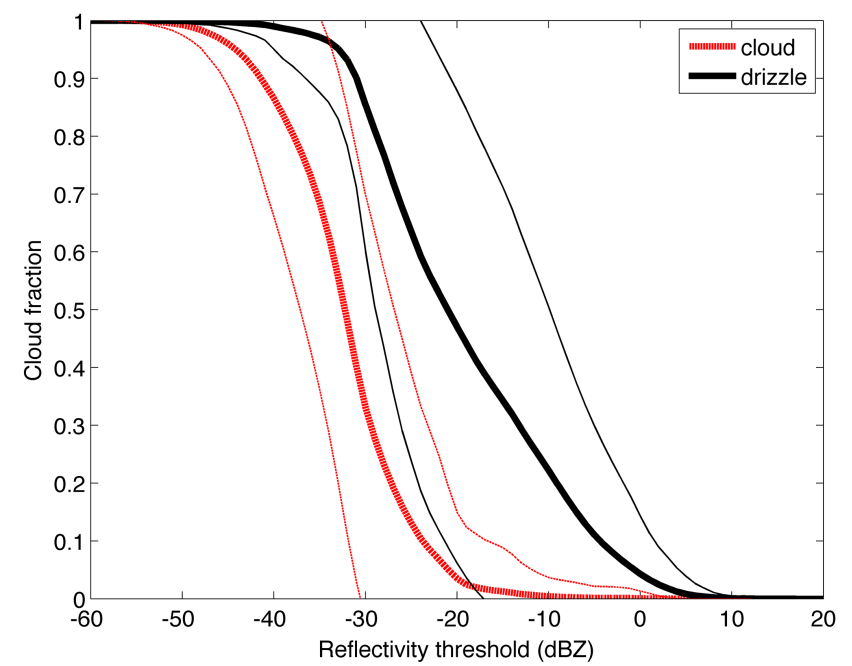

Fig. 2. The radar-detected cloud fraction (mean (thick lines) and standard deviation (thin lines)) for boundary layer clouds at the Azores during the CAP-MBL deployment shown as a function of the radar sensitivity (threshold). Two categories of boundary layer clouds are shown: drizzle-free clouds (red lines) and drizzling clouds (black lines).

clouds cases (a total of $600 \mathrm{~h}$ ) observed by the WACR (Table 1) during the Cloud, Aerosol and Precipitation in the Marine Boundary Layer (CAP-MBL) field experiment (Rémillard et al., 2012) are used in this figure. The marine stratocumulus observations are classified as periods with no radardetected drizzle when the radar-detected echo base is less than $100 \mathrm{~m}$ below the ceilometer-detected cloud base height and as periods with radar-detected drizzle otherwise. The above classification is concerned with drizzle below cloud base. It however does not exclude the presence of drizzle particles above the cloud base; nevertheless, it is used to separate the data set into drizzling and non-drizzling periods. For each class and each hour of observations, the radar-derived hourly cloud fraction (number of columns containing at least one range gate populated by cloud relative to the total number of columns observed) is estimated for different levels of radar sensitivity. It is clear that radar sensitivity plays a critical role in the observed cloud fractions. For a radar sensitivity of $-40 \mathrm{dBZ}$ or better, most stratocumulus clouds are detected (cloud fraction near $100 \%$ ). As the radar sensitivity is degraded (from -40 to $-20 \mathrm{dBZ}$ ), there is a very rapid change in the measured cloud fraction $(\sim 8 \%$ per $1 \mathrm{dBZ}$ drop in sensitivity). Almost none of the clouds are detected for radar sensitivity lower than $-20 \mathrm{dBZ}$. Drizzling clouds have higher reflectivities and thus their cloud fraction is more resilient to changes in radar sensitivity. Similar plots can be constructed at all ARM sites. For instance, at the ARM SGP, shallow cumuli represent an even more challenging target to detect. In contrast, at the ARM northern sites, the presence of highly reflecting ice particles for most of the year decreases the dependency on the radar sensitivity. For reference, the $\mathrm{Ka}-\mathrm{SACR}$ has an estimated sensitivity of $-50 \mathrm{dBZ}$ at $1 \mathrm{~km}$ $(-30 \mathrm{dBZ}$ at $10 \mathrm{~km})$ during nominal operational setting.

\subsection{Radar interpolation schemes}

For weather radars, the volumetric radar observations collected within five minutes are considered instantaneous. Commonly used schemes for gridding such observations to 3-D Cartesian space are the distance-dependent weighted averaging schemes based on the Cressman (1959) and Barnes (1964) weighting functions (Nelson, 1980; Askelson, 1996). Other applied schemes are those based on the nearestneighbor method (Jorgensen et al., 1983) and on bilinear interpolation (Mohr and Vaughan, 1979; Miller et al., 1986; Fulton, 1998). Trapp and Doswell III (2000) analyzed the various theoretical aspects of these schemes and recommended that both the choice of interpolation scheme and the choice of weighting function parameters should be problem specific. They showed that the root-mean-square (rms) differences are smaller in the case of "adaptive" (weighting function defined relative to grid spacing) rather than in the case of "traditional" (fixed weighting function parameters) Cressman and Barnes methods. The "traditional" " Barnes and Cressman methods perform the best in preserving both amplitude and phase of observables, while the errors in amplitude in "adaptive" methods increase with increasing range and data point spacing. Bilinear and nearestneighbor methods generate noise due to smaller-scale wavelengths not present in the initial field and are inferior to the interpolation methods mentioned above, although rms errors between the analyses and observations are the smallest. Zhang et al. (2005) evaluated several gridding methods in order to find the best interpolation approach that retains physical characteristics of raw reflectivity with minimum smoothing or introduction of artifacts on high spatial $(\leq 1 \mathrm{~km})$ and temporal resolution ( $\leq 5 \mathrm{~min}$ ) for real-time applications. The analyzed methods are radar beam volume mapping (a grid cell is considered to be within a radar bin if the center of the grid cell is contained inside the volume of the radar bin), nearest-neighbor method, vertical interpolation method and horizontal and vertical interpolation method. They found that the most suitable method is a scheme combining a nearestneighbor mapping on the range and azimuth plane and a linear interpolation in the elevation direction. Also, a vertical interpolation is preferred for analyses of convective-type echoes, while vertical and horizontal interpolations are recommended for analyses of stratiform echoes.

\subsection{CW-gridding algorithm}

The gridding of Ka-SACR CW-RHI observations is subject to three important facts: the time duration and geometry of the scans as well as the space-time overlap between scans. The elevation scan rate of the Ka-SACR is set to 9 degrees 

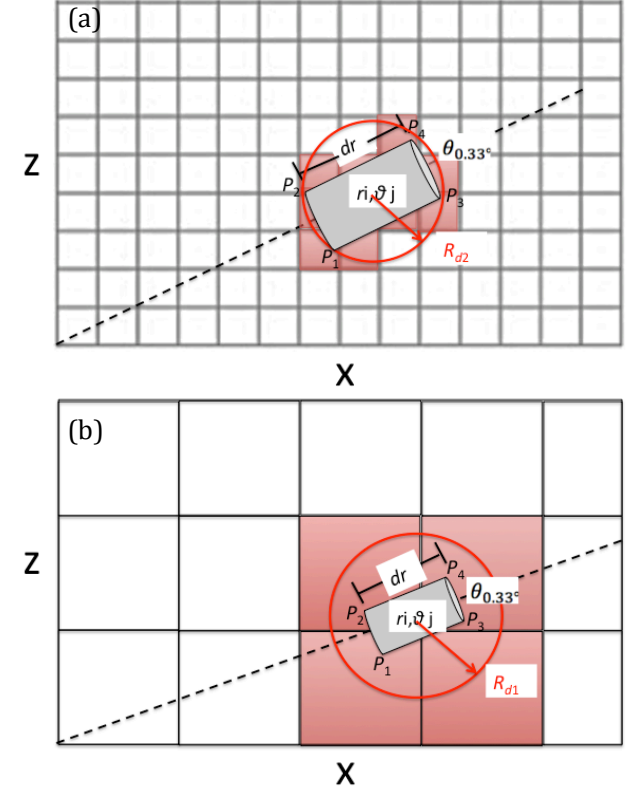

Fig. 3. Vertical cross section of radar resolution volume with fixed azimuth. Identified are the beam width $\left(\theta_{3 \mathrm{~dB}}\right)$, the pulse length $(\mathrm{dr})$, the center of the radar volume in polar coordinates $\left(r_{i}, \theta_{j}\right)$, the edges of radar volume in polar coordinates $\left(P_{1-4}\right)$, the radius of influence ( $R_{d 1}$ and $R_{d 2}$ ) and the influenced grid cells (shaded red). (a) represents cases where the radar observation area is larger than the grid cells and (b) represents cases where the radar observation area is smaller than the grid cells.

per second; thus, it takes about $20 \mathrm{~s}$ to complete a horizonto-horizon scan. A sequence of 60 of such 2-D scans represents a complete set of CW-RHIs (Fig. 1b). The Ka-SACR beamwidth is 0.33 degrees and the radar performs signal integration and records a profile of radar observables every 0.33 degrees of elevation change; thus, it provides a complete coverage with no gaps in the range-height plane. The Ka-SACR produces 26-27 range-resolved measurements of $30 \mathrm{~m}$ resolution (Table 1).

\subsubsection{2-D-gridding algorithm}

The polar-coordinate Ka-SACR observations are first gridded in a height-along-scan distance 2-D Cartesian grid and are then interpolated in time. At $100 \mathrm{~m}$ range from the KaSACR, the shortest distance between centers of radar resolution volume at two adjacent elevations (the chord length) is $0.5 \mathrm{~m}$, while at $15 \mathrm{~km}$ range it is $80 \mathrm{~m}$. Thus, at short range, the radar observations in a contiguous set of elevations will influence the values in a particular grid point more, while at far range the radar observations along the range will have a stronger influence on the grid point value. The degree of smoothing effects depends on the chosen resolution in Cartesian space. The chosen gridding method thus has to be based on adaptive weights depending on both the size of the radar

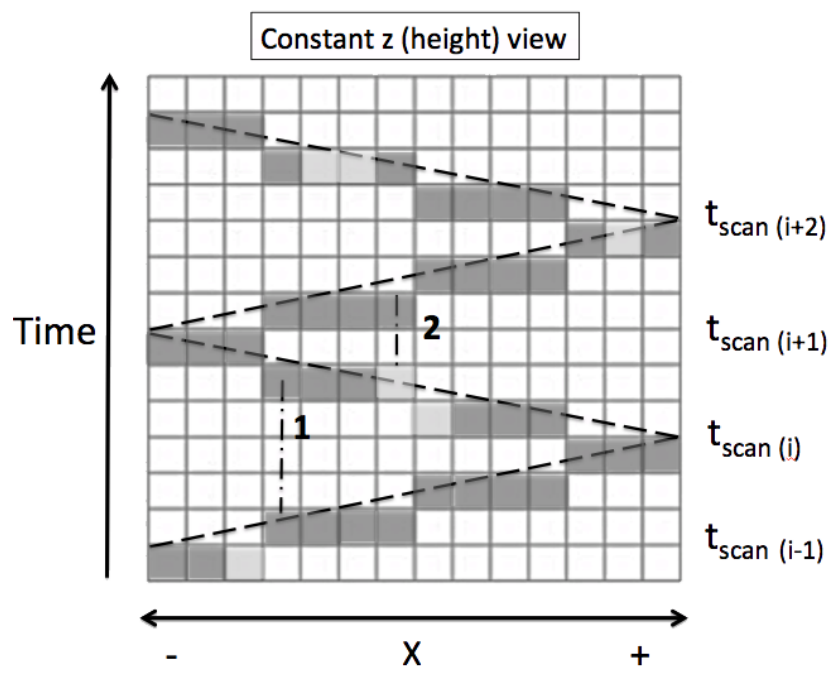

Fig. 4. Schematic of the consecutive CW-RHI scans at constant height. Scenario 1: neighboring scans contain cloud (dark squares). Scenario 2: previous scan does not contain cloud (light squares) and the next scan contains cloud.

volume (in order to minimize the smoothing) and resolution in Cartesian space.

The polar $(r, \varphi, \theta)$ to Cartesian $(x, y, z)$ transformation is performed for each individual cloud pixel observed. The standard atmospheric beam propagation model (Doviak and Zrnić, 1993) is assumed in all computations:

$a_{\mathrm{ef}}=\frac{4}{3} a$,

$z=\left(r^{2}+a_{\mathrm{ef}}^{2}+2 r a_{\mathrm{ef}} \sin \theta\right)^{0.5}-a_{\mathrm{ef}}$,

$s=a_{\mathrm{ef}} \sin ^{-1}\left(\frac{r \cos \theta}{a_{\mathrm{ef}}+z}\right)$,

where $a$ represents the Earth's radius, $a_{\text {ef }}$ represents the 4/3effective Earth radius, $z$ is the height of the center of the radar resolution volume, and $s$ is the distance between the radar and the projection of the bin along the earth surface. The radar coordinates $(r, \varphi, \theta)$ represent the slant range, azimuth angle following mathematical convention and elevation angle from the horizon, respectively. The horizontal Cartesian coordinates $x$ and $y$ are then determined by

$x=s \cos \phi$,

$y=s \sin \phi$.

Setting the azimuth angle to 0 degrees performs the transition from 3-D to the range-height plane imposed by the CW scanning strategy. The effects of Earth curvature are included in 
the coordinate transformation equations although the targetplane approximation is appropriate for practical applications of CW-RHI radar data.

The Ka-SACR sampling strategy is such that the angle difference between two successive elevations is equal to the antenna 3 degree beamwidth, $\theta_{0.33^{\circ}}$. In reality, and due to reasons associated with small fluctuations of the scanner rotational velocity and the real-processing computer efficiency, the angle difference between two successive elevations may differ from $\theta_{0.33^{\circ}}$ so that somewhat small gaps between the adjacent elevations may exist. Analysis of a large volume of Ka-SACR observations indicated that, typically, the angle is seldom wider than two times the $\theta_{0.33^{\circ}}$. For the sake of simplicity, the antenna beamwidth in azimuthal direction is not taken into account in the remaining discussion.

Radar observations centered on the polar coordinate $r_{i}, \theta_{j}$ are considered to be representative of the area defined by the range $r_{i}, \theta_{0.33^{\circ}}$, and pulse length $\mathrm{dr}$ with polar coordinates $\left[P_{1}=\left(r_{i}-\mathrm{dr} / 2, \theta_{i}-\theta_{0.33^{\circ}} / 2\right), P_{2}=\left(r_{i}-\mathrm{dr} / 2, \theta_{i}+\theta_{0.33^{\circ}} / 2\right)\right.$, $\left.P_{3}=\left(r_{i}+\mathrm{dr} / 2, \theta_{i}-\theta_{0.33^{\circ}} / 2\right), P_{4}=\left(r_{i}+\mathrm{dr} / 2, \theta_{i}+\theta_{0.33^{\circ}} / 2\right)\right]$ (Fig. 3). The radar resolution area increases with increasing distance from the radar, and thus it is necessary to have a variable radius of influence. In some cases, the radar observation area is larger than the grid cell area; thus the radar observation area contains grid cells (within the radius of influence $R_{d 2}$, Appendix A1). The algorithm estimates the polar coordinates for all grid points and only those grid pixels inside of the radar area $r_{i}, \theta_{j}$ (bounded by polar coordinates $P_{1-4}$ ) are considered to have a value influenced by the observational value $r_{i}, \theta_{j}$. In other cases (for instance at small distances from radar or for large grid resolutions), the radar observation area is very small compared to the grid cell area and it may not contain any grid points. In those cases, the observational value $r_{i}, \theta_{j}$ is considered to influence those grid points located within the grid resolution (radius $R_{d 1}$, Appendix A). Each grid point is allowed to "have a memory" of all "influencing" observational points and their distance from the grid point. Finally, once each radar resolution volume is examined, and the observational values that influence each specific grid point are identified, one of the following gridding methods is applied to estimate the radar value at the grid point: maximum value, mean value, Cressman or Barnes (see Appendix A for details on the available gridding methods).

\subsubsection{Along-time gridding algorithm}

Once the Ka-SACR polar coordinate observations are gridded in 2-D range-height planes, the gridded planes are interpolated in time (Fig. 4). The selected temporal resolution $\Delta t$ is preferred to be greater than or equal to the average time between two successive scans (about $1 \mathrm{~s}$ for the Ka-SACR CW-RHI scans). Furthermore, $\Delta t$ should not be higher than a quarter of the scan duration. A schematic of the scanning space at constant height $z$ along the time dimension is shown in Fig. 4. The dashed lines represent a few consecutive scans

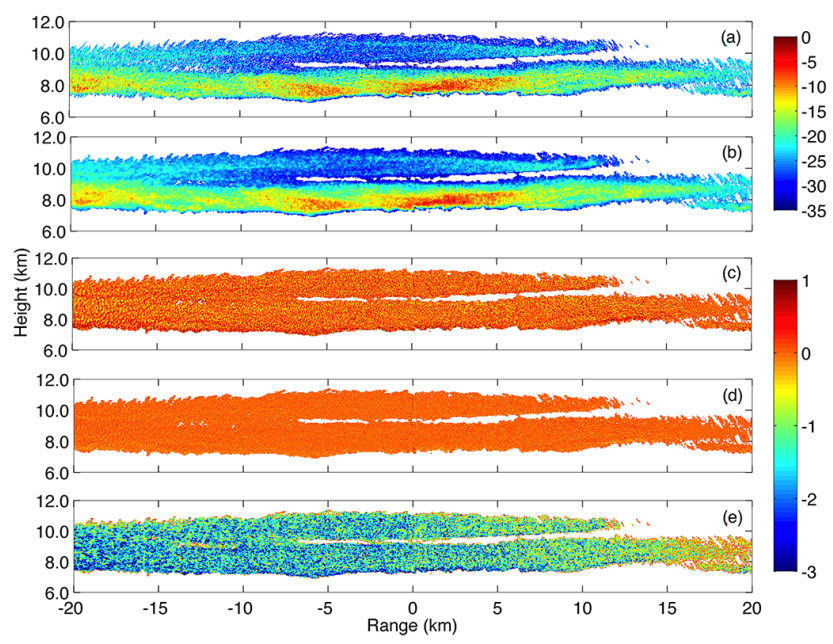

Fig. 5. Ka-SACR radar reflectivity 2-D gridding ( $\Delta x=50 \mathrm{~m}, \Delta z=$ $50 \mathrm{~m}$ ) for a thick cirrus cloud observed at the ARM SGP site: (a) polar coordinates $(r, \theta)$ Ka-SACR post-processed data; (b) $x$ - $z$ gridded radar reflectivity using the Barnes scheme; (c) difference in gridded radar reflectivity between the Barnes and Cressman schemes $\left(\mathrm{dBZ}_{\mathrm{BARNES}}-\mathrm{dBZ}_{\mathrm{CRESSMAN}}\right)$; (d) difference in gridded radar reflectivity between the Barnes and mean value schemes; and (e) difference in gridded radar reflectivity between the Barnes and maximum value schemes.

along the scanning direction $x$ that appear tilted in time since each horizon-to-horizon scan required a finite time period to be completed $(20 \mathrm{~s})$. The grid resolution along the crosswind direction as well as the temporal resolution (density of grid points along the time dimension) is user-dependent. First, using the nearest-neighbor method only along the time dimension, grid points are populated with the 2-D gridded values. The locations of these $\left(x_{\mathrm{ev}}, z, t_{\mathrm{ev}}\right)$ points are denoted by the shaded squares near the dashed lines in Fig. 4 and represent our best estimates of the gridded CW-RHI scanning radar observations, denoted by the expression "measured values" in the remainder of the text.

The "measured values" are not regularly distributed along the time coordinate for different fixed positions at the scanning direction ( $x$ axis). The time distance between the measured points varies between the average time between two successive scans and twice the time duration of the scan. For example, at the center of the grid $(x=0)$, the distance between any two measured points is approximately $21 \mathrm{~s}$, while at the furthest edges of $x$ it varies between 1 and $41 \mathrm{~s}$. The number of grid points between any two measured values thus varies as a function of grid resolution in time direction and properties of scanning technique. The values at these points are obtained using the two closest measured values at $\left(x_{\mathrm{ev}}, z_{\mathrm{ev}}\right)$, that is, the one from the preceding and the one from the following scan. If the two measured values contain radar detections (case 1, Fig. 4), then linear interpolation is performed for all the points between the two measured values. 

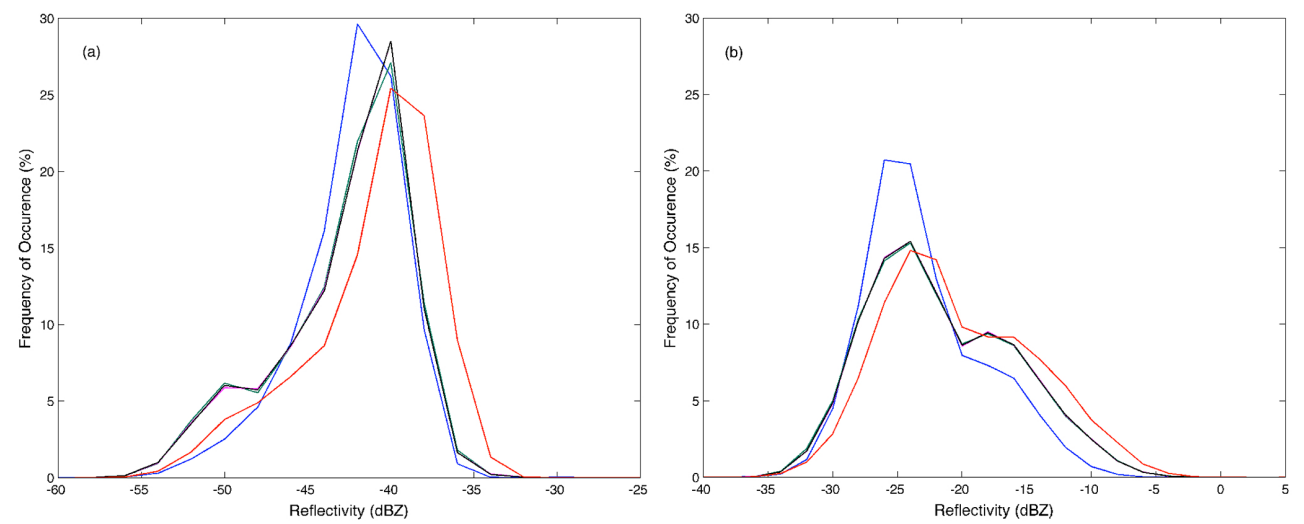

Fig. 6. Area distribution histograms of Ka-SACR radar reflectivity 2-D gridding for (a) a stratus cloud $(\Delta x=25 \mathrm{~m}, \Delta z=25 \mathrm{~m})$ and (b) a cirrus cloud $(\Delta x=50 \mathrm{~m}, \Delta z=50 \mathrm{~m})$. The radar reflectivity histograms are shown for the polar coordinates Ka-SACR post-processed data (blue) and for all interpolation schemes (Cressman: green; Barnes: black; maximum: red; and mean: magenta).

If one of the two measured values contains no radar detection (case 2, Fig. 4), then each measured value is assumed constant and is propagated in half of the points, that is, only to the start time of the following and/or the end-time of the preceding scan.

\subsubsection{Evaluation of the gridded SACR radar observables}

A cirrus cloud sampled by the SGP Ka-SACR is used as an example to investigate the performance of the 2-D gridding algorithm and the influence of the selected interpolation scheme on the gridded radar reflectivity values (Fig. 5). The cirrus cloud is clearly detected over a $40 \mathrm{~km}$ range and has a double layer structure with cloud base at $7-8 \mathrm{~km}$ and cloud top at $10-11 \mathrm{~km}$ as mapped by the polar coordinates Ka-SACR post-processed data (Fig. 5a). The 2-D Cartesiangridded $(\Delta x=50 \mathrm{~m}, \Delta z=50 \mathrm{~m})$ field of Ka-SACR reflectivities using the Barnes scheme is a bit smoother than expected but all important features and scales of variability are preserved (Fig. 5b). The 2-D gridded radar reflectivity differences between the Barnes scheme and the Cressman, mean value and the maximum value interpolation schemes are shown in Fig. 5c-e. Very small differences are observed between the Barnes and the Cressman and mean value interpolation schemes. The maximum value interpolation scheme results in higher overall reflectivity values compared to the Barnes scheme and subsequently compared to the Cressman and mean value schemes. Near the cirrus cloud edges and in areas with low radar reflectivity values (at ranges 15-20 km), the differences between the maximum value scheme and the Barnes scheme are smaller. Their larger similarity in those areas is due to a lack of reflectivity variability due to lower radar sensitivity.

Another method to evaluate the performance of the various interpolation schemes consists of comparing the area distribution histograms of Ka-SACR reflectivities before and after gridding using all available interpolation schemes (Fig. 6). This is done for two cases: a cirrus cloud observed at the SGP and a stratus cloud observed at TCAP. The stratus cloud is characterized by lower radar reflectivities with a distribution peak at $-42 \mathrm{dBZ}$ (Fig. 6a). Due to its very low radar reflectivities the stratus cloud is not detected at ranges extending farther than $5 \mathrm{~km}$ from the Ka-SACR. The cirrus cloud is characterized by higher radar reflectivities with a distribution peak at $-25 \mathrm{dBZ}$ (Fig. 6b) and it is detected at ranges up to $20 \mathrm{~km}$ (Fig. 5a). Unsurprisingly, the maximum value interpolation scheme shifts the area distribution histogram of reflectivities to values $3 \mathrm{~dB}$ higher than compared to before gridding. Furthermore, the area distribution histograms of the Cressman, Barnes and mean value interpolation schemes are very similar in both cases. Overall, there is reasonable agreement between the histograms of the three interpolation schemes and the Ka-SACR post-processed data. However, it is noticeable that in the case of the stratus cloud, the interpolation schemes produce a small bump (increase) in the areal coverage of lower reflectivities. This is imputable to the weak radar reflectivities of the non-precipitating stratus cloud that results in areal gaps near cloud edges that are filled with low radar reflectivity values during gridding. In the case of the cirrus cloud, a small increase in the areal coverage of low radar reflectivities is also observed, but it is much smaller due to the relatively insignificant areal coverage of cloud edges compared to the thick and horizontally extensive cirrus area. On the other hand, the interpolation schemes produce a small bump in the areal coverage of high reflectivities, which can be attributed to the smoothing effect of the interpolation schemes (Fig. 6b).

An example of along-time gridding at a constant height of $7175 \mathrm{~m}$ near the base of a cirrus cloud observed at the ARM SGP site is shown in Fig. 7. The location and density of the measured values are shown in the 2-D cloud mask (black areas in Fig. 7a). The location of the interpolated points 


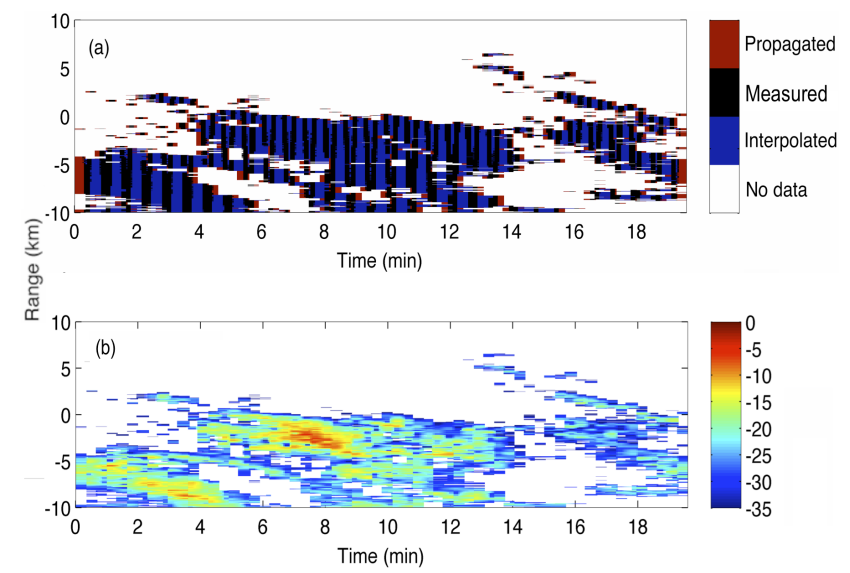

Fig. 7. Ground-parallel cross section (constant height) and $x$ axis along the scanning direction of a cirrus cloud observed at the SGP: (a) the cloud mask of the 3-D gridded data, and (b) the 3-D gridded Ka-SACR radar reflectivity field.

(i.e., cloud pixels located between measured values located at preceding and subsequent scans) is shown (blue areas in Fig. 7a). Finally, the location of the propagated points (i.e., cloud pixels located between one missing and one present measured value) is shown (red areas in Fig. 7a). The corresponding Ka-SACR gridded reflectivity field is shown in Fig. $7 b$.

The observations from the collocated dedicated ARM profiling radars produce continuous high-resolution time-height observations and a method to (i) evaluate the performance of the along-time gridding algorithm and (ii) investigate whether the temporal spacing between the CW-RHI scans (currently $20 \mathrm{~s}$ over the radar location) is sufficient to adequately capture cloud-scale variability. A comparison between the observed (post-processed) and the gridded KaSACR extracted at zenith and the high-resolution KAZR observations is shown in Fig. 8. The comparison is limited to the radar reflectivity field for a SGP cirrus. The ability (sensitivity) of the two radar systems to capture the structure of the thick cirrus layer is comparable. Since the Ka-SACR uses shorter integration times compared to the KAZR (high-power Klystron transmitter compared to the low output travelingwave tube), it allows comparable sensitivity performance. As expected, the KAZR observations provide a very highresolution mapping of the cirrus cloud structure (Fig. 8c). The post-processed Ka-SACR reflectivity (Fig. 8b) observations are of much lower temporal resolution $(20 \mathrm{~s}$ vs. $2 \mathrm{~s}$ for the KAZR). The temporal resolution of the $\mathrm{CW}$-gridded KaSACR reflectivities (Fig. 8a) is set to $3 \mathrm{~s}$ in this case and provides a smoother field of radar reflectivity that compares well with the KAZR reflectivity field. A comparison of the time series of the radar reflectivity at a specific height (shown by the black line in Fig. 8c) indicates that the Ka-SACR gridded observations that are based on the Barnes interpolation

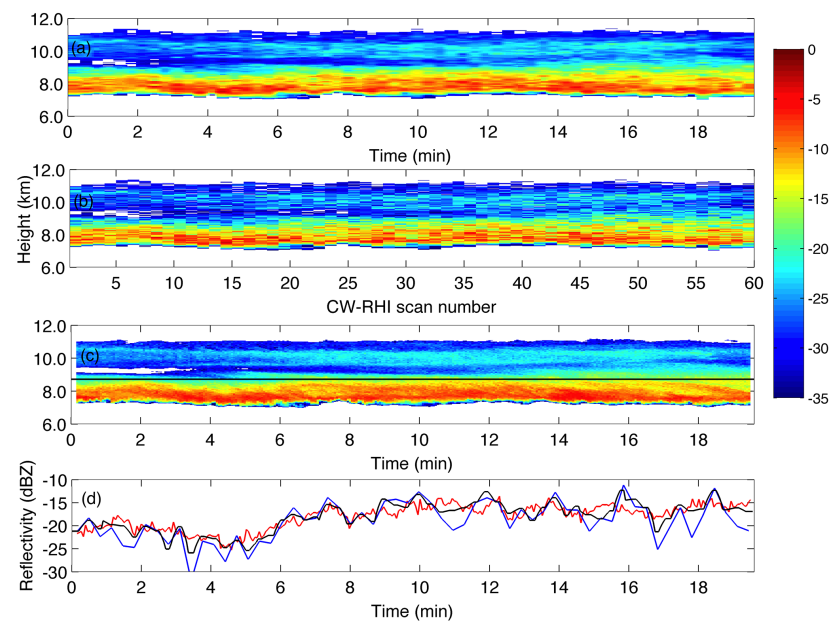

Fig. 8. Time-height radar reflectivity field of a cirrus observed at the SGP with the (a) Ka-SACR gridded $(x=0 \mathrm{~m})$, (b) Ka-SACR postprocessed polar coordinates $\left(\theta=0^{\circ}\right)$, and (c) profiling KAZR. The black line indicates the height of panel (d) retrievals. (d) Time series of radar reflectivity from Ka-SACR gridded ( $x=0 \mathrm{~m}$, black), KaSACR post-processed polar coordinates $\left(\theta=0^{\circ}\right.$; blue) and profiling KAZR (red).

scheme agree better with the KAZR high-resolution measurements (Fig. 8d).

A similar comparison of the vertical Doppler velocity field is shown for a low-level stratus cloud observed at TCAP (Fig. 9). This comparison is more challenging since boundary layer clouds usually have a turbulent vertical velocity field (Kollias and Albrecht, 2000). It is important to note that the Ka-SACR has a dead zone of more than $600 \mathrm{~m}$ due to the use of pulse compression mode to enhance its sensitivity. Thus, the Ka-SACR observations miss a part of the cloud near the cloud base that the vertically pointing WACR observed (Fig. 9c). Starting with the cloud boundaries, it is clear that both the post-processed and the gridded KaSACR observations capture the cloud-top height variability observed by the high-resolution WACR. The WACR cloudtop height is higher, demonstrating the higher sensitivity of the WACR. The WACR Doppler velocity observations reveal a sequence of coherent updraft and downdraft structures. In this particular example, the processed Ka-SACR Doppler velocities extracted at zenith pointing (Fig. 9b) resolve the coherent dynamical structures. In general, the ability of the Ka-SACR to resolve dynamical structures in boundary layer clouds will depend on the horizontal wind speed and the horizontal scale of the in-cloud vertical motions. The gridded Ka-SACR Doppler velocity field provides a smoother field (Fig. 9a). Note that in the gridded field the interpolation has horizontally stretched the dynamical structures; however, their magnitude is maintained and no artifacts have been introduced. This fact is reflected in the overall good agreement between the gridded field and the high-resolution 


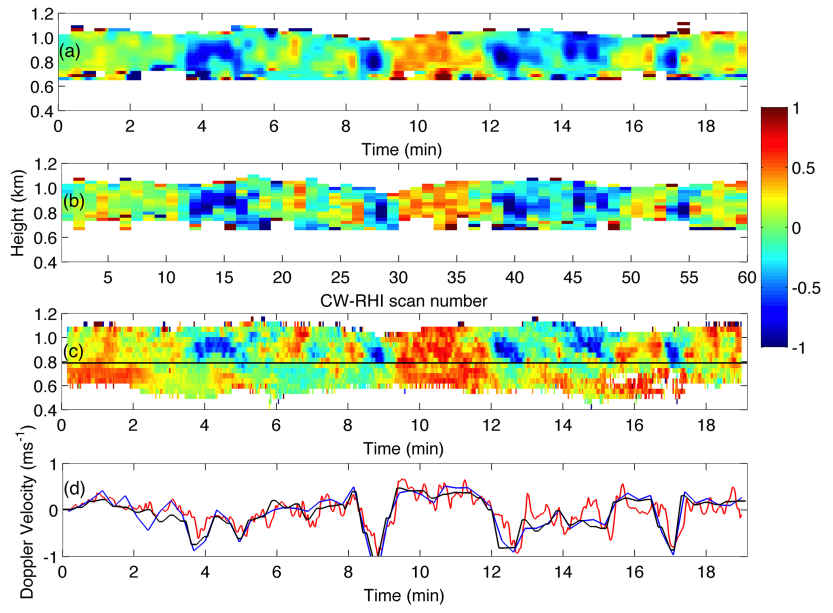

Fig. 9. Time-height radar Doppler velocity field of a stratocumulus observed at TCAP with the (a) Ka-SACR gridded $(x=0 \mathrm{~m})$, (b) Ka-SACR post-processed polar coordinates $\left(\theta=0^{\circ}\right)$, and (c) profiling KAZR. The black line indicates the height of panel (d) retrievals. (d) Time series of radar reflectivity from Ka-SACR gridded ( $x=0 \mathrm{~m}$, black), Ka-SACR post-processed polar coordinates $\left(\theta=0^{\circ}\right.$; blue) and profiling KAZR (red).

WACR observations. A comparison of the time series of the Doppler velocity at a specific height (shown by the black line in Fig. 9c) confirms that the Ka-SACR gridded observations capture most of the variance of the Doppler velocity field as observed by the WACR (Fig. 9d).

\section{Retrieval of vertical Doppler velocity component from radial Doppler velocity measurements}

The vertical Doppler velocity $\left(V_{\mathrm{D}, \mathrm{V}}\right)$ in clouds is the sum of the vertical air motion $\left(w_{\text {AIR }}\right)$ and the reflectivity-weighted particle fall velocity $\left(V_{\mathrm{F}}\right)$. Depending on the relative contribution of the two terms, the $V_{\mathrm{D}, \mathrm{V}}$ can be used to estimate updraft mass flux and high-order vertical air motion statistics (Kollias and Albrecht, 2000) as well as for aerosol activation studies near the cloud base and for particle size retrievals (Frisch et al., 1995; Deng and Mace, 2006). Scanning cloud radars measure the radial Doppler velocity $\left(V_{\mathrm{D}, \theta}\right)$ component that is composed of the projected horizontal wind velocity parallel to the RHI scan plane $\left(V_{\mathrm{H}, \mathrm{RHI}}\right)$ and the projected $V_{\mathrm{D}, \mathrm{V}}$, as depicted by Eq. (6) and illustrated by Fig. 10.

$V_{\mathrm{D}, \theta}(\theta)=V_{\mathrm{H}, \mathrm{RHI}} \cos (\theta)+V_{\mathrm{D}, \mathrm{V}} \sin (\theta)$,

where $\theta$ is the elevation angle. At the two extreme elevation angles, vertically $\left(\theta=90^{\circ}\right)$ and horizontally $\left(\theta=0^{\circ}\right)$, the radial Doppler velocity is dominated by $V_{\mathrm{D}, \mathrm{V}}$ and $V_{\mathrm{H}, \mathrm{RHI}}$ respectively. Thus, the zenith measurements from the SACR or the collocated profiling cloud radar can serve as a reference for the order of magnitude of the vertical velocity retrievals.

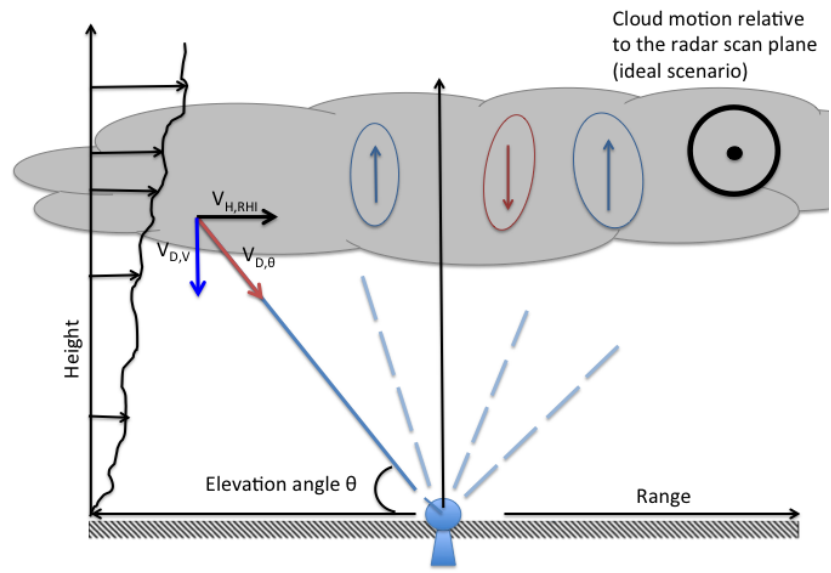

Fig. 10. Schematic of Ka-SACR CW-RHI Doppler velocity observations. Identified are the elevation angle $(\theta)$, the horizontal wind projection on the radar scan plane $\left(V_{\mathrm{H}, \mathrm{RHI}}\right)$, the vertical Doppler velocity $\left(V_{\mathrm{D}, \mathrm{V}}\right)$ and the observed angular Doppler velocity $\left(V_{\mathrm{D}, \theta}\right)$.

Adversely, as the radar beam moves away from zenith, the horizontal wind contribution $\left(V_{\mathrm{H}, \mathrm{RHI}} \cos (\theta)\right)$ increases, tracing a sinusoidal pattern (velocity elevation display, VED) similar to the cosine pattern seen in the classic velocity azimuth display (VAD; Lhermitte and Atlas, 1961; Browning and Wexler, 1968). If $V_{\mathrm{H}, \mathrm{RHI}}$ is known with reasonable accuracy, then a best estimate of the vertical Doppler velocity component can be retrieved.

\subsection{Estimation of the horizontal wind component parallel to the RHI scan plane $V_{\mathbf{H}, \mathrm{RHI}}$}

At the ARM sites, a total of two to four soundings are launched daily to provide profiles of the thermodynamic structure of the atmosphere. However, considerable variability of the horizontal wind profile is expected between the 6 to $12 \mathrm{~h}$ gaps, especially in the boundary layer. Furthermore, the retrieved quantity $V_{\mathrm{D}, \mathrm{V}}$ has small magnitudes in clouds; thus, it is important to have an accurate knowledge of the wind field. The magnitude of $V_{\mathrm{F}}$ varies from negligible in the case of cloud droplets to $0.5-2.0 \mathrm{~m} \mathrm{~s}^{-1}$ for drizzle and ice/snow particles and up to $4.0-9.0 \mathrm{~m} \mathrm{~s}^{-1}$ in the case of rain (at reference surface conditions). The technique is applicable to clouds and precipitation with variable $V_{\mathrm{F}}$ since our objective is to retrieve the best estimate for $V_{\mathrm{D}, \mathrm{V}}$ and not to decompose $V_{\mathrm{D}, \mathrm{V}}$ into its components. The application of the technique is limited to cloud systems with a negligible horizontal gradient of $V_{\mathrm{H}, \mathrm{RHI}}$ over the range of 10 to $20 \mathrm{~km}$. In effect, it is applicable to cloud systems that do not alter the environmental wind flow in any significant way (e.g., deep convective clouds). Another limitation of the technique is the need to have hydrometeor detections at a particular height on each side of the radar and over a range of elevation angles to minimize the uncertainty in the retrieved $V_{\mathrm{H}, \mathrm{RHI}}$. Although $100 \%$ hydrometeor fractional coverage is not required to retrieve a 

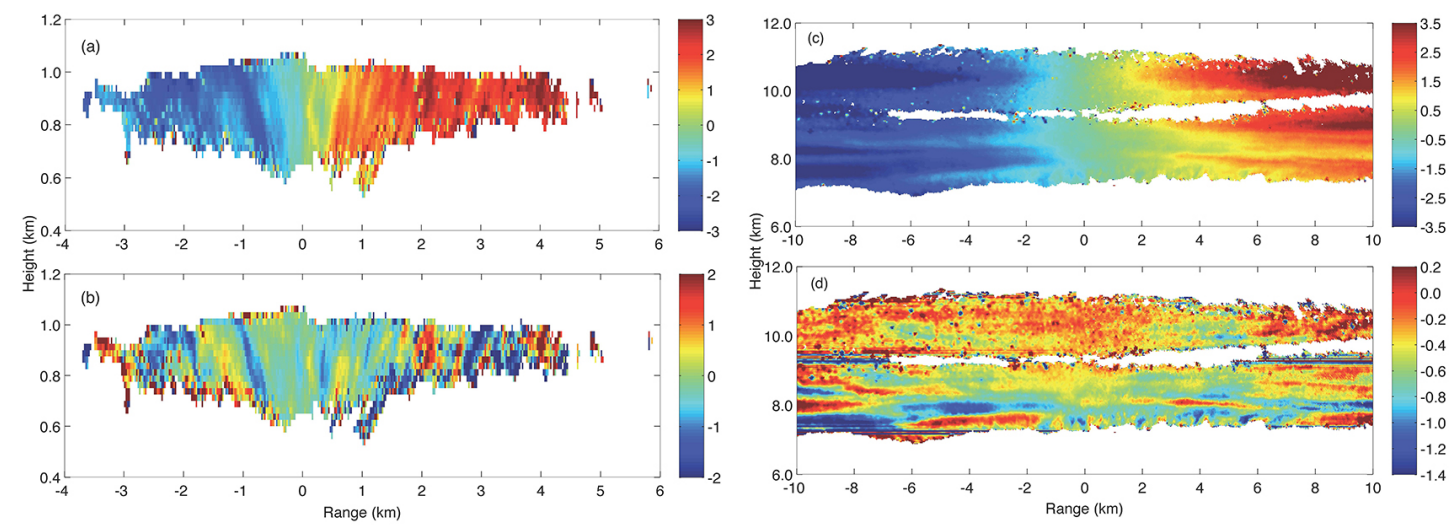

Fig. 11. Ka-SACR radar Doppler velocity 3-D gridding RHI planes of a stratocumulus during TCAP: (a) observed radial Doppler velocity and (b) retrieved vertical Doppler velocity component; Ka-SACR radar Doppler velocity 3-D gridding RHI planes of a cirrus at the SGP: (c) observed radial Doppler velocity and (d) retrieved vertical Doppler velocity component.

$V_{\mathrm{H}, \mathrm{RHI}}$, a large number of points will reduce the impact of the $V_{\mathrm{D}, \mathrm{V}}$ variability on the retrievals.

The input to the $V_{\mathrm{D}, \mathrm{V}}$ retrieval technique consists of the 2-D CW-gridded RHI planes. The retrievals are performed at each gridded height with hydrometeor detections and for all the 2-D gridded planes that compose a 20 min set $(380$ for $\Delta t=3 \mathrm{~s}$ ). In principal, the technique used is similar to the VAD analysis (Browning and Wexler, 1968) with a few modifications that are discussed below. First, at each constant height all $V_{\mathrm{D}, \theta}$ observations at elevations $75-105^{\circ}$ are averaged to produce a first estimate of the average value of $\left\langle V_{\mathrm{F}}\right\rangle$. Subsequently, all $V_{\mathrm{D}, \theta}$ observations at elevations lower than $75^{\circ}$ or higher than $105^{\circ}$ are used to produce estimates of $V_{\mathrm{H}, \mathrm{RHI}}$ at each elevation using the following formula:

$$
\begin{aligned}
V_{\mathrm{H}, \mathrm{RHI}}(\theta) & =\frac{V_{\mathrm{D}, \theta}(\theta)-\left\langle V_{\mathrm{F}}\right\rangle \sin (\theta)}{\cos (\theta)} \\
& \left(\theta<75^{\circ} \text { or } \theta>105^{\circ}\right) .
\end{aligned}
$$

Rather than estimating the mean and the standard deviation of the $V_{\mathrm{H}, \mathrm{RHI}}(\theta)$, a linear model of $V_{\mathrm{H}, \mathrm{RHI}}(x)$ is fitted to the data $\operatorname{set}\left(x, V_{\mathrm{H}, \mathrm{RHI}}\left(x=z \tan (\theta)^{-1}\right)\right.$ :

$V_{\mathrm{H}, \mathrm{RHI}}(x)=\beta+\alpha x$.

The intercept $\left(\beta, \mathrm{m} \mathrm{s}^{-1}\right)$ represents the bulk of $V_{\mathrm{H}, \mathrm{RHI}}$ and the slope $\left(\alpha, \mathrm{s}^{-1}\right)$ is introduced to quantify small linear trends. The estimation of a linear fit rather than the direct estimation of the mean is based on the analysis of a large data set of Ka-SACR CW-RHI observations that have demonstrated the presence of small horizontal wind variations across the Ka-SACR scan plane. Considering that several of the SACR systems are mobile, the use of the linear fit is appropriate to account for horizontal wind variations related to topographic influences, sea-land contrast, etc. The parameters $\alpha$ and $\beta$ are estimated at each gridded height and for each rangeheight plane. The smoothness of the field of the parameters $\alpha$ and $\beta$ is used to evaluate the robustness of the proposed technique.

Subsequently, Eq. (6) is used to retrieve the best estimate of the vertical Doppler velocity at each height and for each range-height planes. Figure 11 shows two examples of processed Ka-SACR Doppler velocities and the corresponding retrieved vertical Doppler velocities. The stratus cloud observations were recorded at TCAP and the cirrus cloud observations were recorded at the SGP. It is apparent that the processed Doppler velocity fields (Fig. 11a, c) are of little scientific use in the raw format. Their values depend on the combination of the atmospheric wind profile and the Ka-SACR azimuth and elevation angle. The retrieved vertical Doppler velocity fields (Fig. 11b, d) are not influenced by the two aforementioned factors and are thus more related to vertical dynamics and particle sedimentation. In the case of the stratus cloud, there is no drizzle; thus, the observed vertical Doppler velocity structures are manifestations of vertical air motion drafts. In the cirrus case, particle sedimentation $\left(V_{\mathrm{F}}\right)$ is nonnegligible. The retrieved vertical Doppler velocity field is a combination of vertical dynamics (e.g., gravity waves) and particle sedimentation. In both cases, the retrievals are noisy near cloud edges due to the reduced amount of cloud observations.

\subsection{Evaluation of the 3-D vertical Doppler velocity retrievals}

At each height and range, the mean of different Doppler velocity components in time is computed (19 min average). Examples of a stratus and a cirrus are presented in Fig. 12a and $b$ respectively. The observed (post-processed) Ka-SACR Doppler velocities at the middle of the cloud layer (constant height; red lines) and the sinusoidal fit $\left(V_{\mathrm{H}, \mathrm{RHI}} \cos (\theta)\right.$; blue lines) and its offset $\left\langle V_{\mathrm{F}}\right\rangle$ are shown for each case. As expected, $\left\langle V_{\mathrm{F}}\right\rangle$ is negligible in the stratus and $0.3 \mathrm{~m} \mathrm{~s}^{-1}$ in the cirrus. The temporally averaged retrieved vertical velocity 


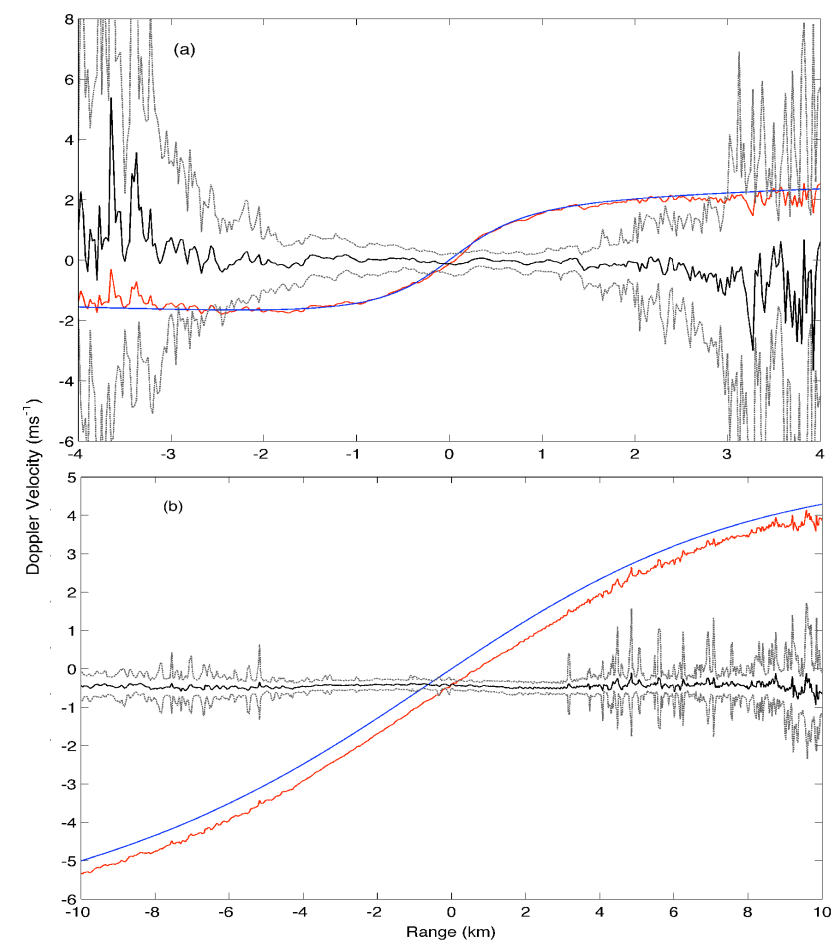

Fig. 12. Nineteen min averaged Doppler velocities at the middle of (a) a stratocumulus cloud layer and (b) a cirrus cloud case as a function of range from the radar. The 19 min average implies that all 3-D gridded CW-RHI planes at this height have been used to estimate the mean and variance of the retrieved Doppler velocities. The radial observed Doppler velocities $\left(V_{\mathrm{D}, \theta}\right)$ are shown in red, the fitted projected horizontal wind contributions $\left(V_{\mathrm{H}, \mathrm{RHI}} \cos (\theta)\right)$ are shown in blue and the retrieved vertical Doppler velocity $\left(V_{\mathrm{D}, \mathrm{V}}\right)$ in black. The standard deviation of the retrieved vertical Doppler velocity is in dashed grey.

component and its variance (solid and dashed black lines) are a good indicator of the quality of the retrieved field at each height and range for all times and can be used as a quality flag for the retrieved vertical Doppler velocity field. Normally, the standard deviation of vertical Doppler velocity measurements is an indicator of in-cloud turbulence intensity. In the case of the stratus layer, the standard deviation is almost constant within $\pm 1.5 \mathrm{~km}$ along the scan range from the radar. Beyond this range, the standard deviation grows fast. Since there is no apparent reason for the cloud to be more turbulent at far ranges, it is plausible to assume that the observed growth in the standard deviation is due to erroneous estimates of $V_{\mathrm{D}, \mathrm{V}}$ at far ranges. In the case of the cirrus layer, the retrievals are stable up to much larger ranges $( \pm 10 \mathrm{~km})$. This difference is due to the fact that at higher heights, larger ranges can be covered with the same elevation angle swath. Note that the enhancement of the vertical Doppler velocity standard deviation for positive range values $(x>+4 \mathrm{~km})$ is associated with weaker radar echoes and more areas with no radar detections in time. The estimates of $V_{\mathrm{D}, \mathrm{V}}$ standard deviation will be included in any future products based on the proposed gridding and retrieval techniques.

Examples of the unique insights into 3-D cloud dynamics provided by the Ka-SACR gridded observations of vertical Doppler velocity are shown in Fig. 13. A ground-parallel cross section near the middle of the clouds as well as a rangeheight cross section in the middle of the scanning period $(10 \mathrm{~min})$ are presented. The stratus cloud layer extends across a $5 \mathrm{~km}$ range as shown in Fig. 13a while the cirrus cloud layer extends across a $10 \mathrm{~km}$ range as illustrated in Fig. 13b. The stratus cloud is characterized by very low radar reflectivity values and no echoes below the cloud base; thus the influence of drizzle particle sedimentation $\left(V_{\mathrm{F}}\right)$ on the observed $V_{\mathrm{D}, \mathrm{V}}$ is negligible. Given this, the $V_{\mathrm{D}, \mathrm{V}}$ field is representative of the vertical air motions ( $\left.w_{\text {AIR }}\right)$ in the cloud layer. The gridded $V_{\mathrm{D}, \mathrm{V}}$ observations clearly indicate the presence of linear, coherent dynamical structures (positive: updrafts; negative: downdrafts) with vertical extent from cloud base to cloud top. Those features also transit faultlessly from positive to negative crosswind ranges which validates the assumption that the component of the horizontal wind has been successfully removed. In the deep cirrus layer, particle sedimentation $\left(V_{\mathrm{F}}\right)$ is not negligible and results in overall negative $V_{\mathrm{D}, \mathrm{V}}$ values with comparable velocities across the range, further supporting the successful removal of the horizontal wind contribution. The horizontal plane of $V_{\mathrm{D}, \mathrm{V}}$ shows low frequency variability that can be the result of the action of gravity waves combined with spatial inhomogeneity in particle sedimentation. The vertical cross section indicates a complicated vertical structure with several vertical layers with different fall velocities.

\section{Summary}

Scanning cloud radars are currently operational at several ground-based supersites around the world. Their observations aim to address the need to (i) describe the 3-D cloudy atmosphere (morphology and dynamics) and (ii) to track and monitor the lifetime of clouds. The Cross-Wind RangeHeight Indicator (CW-RHI), a sequence of scans that slice cloud layers that move over the radar site in a plane perpendicular to that of the cloud layer mean wind direction, is a sampling strategy often used for cloud radars.

Independent of the scan strategy employed, it is apparent that radar-derived cloud fraction $\mathrm{CF}_{\mathrm{Hor}}$ and 3-D hydrometeor boundaries depend on the radar sensitivity that decreases with the square of the range from the radar. Using profiling radar observations of marine boundary layer clouds, the dependency of the measured $\mathrm{CF}_{\mathrm{Hor}}$ on radar sensitivity is demonstrated. In non-drizzling clouds, a drop of the radar sensitivity from -30 to $-20 \mathrm{dBZ}$ can result in a dramatic change in the radar-derived $\mathrm{CF}_{\mathrm{Hor}}$. Similar analyses at different ARM sites and for different cloud systems can be used to (i) assess the low $\mathrm{CF}_{\text {Hor }}$ bias introduced with 

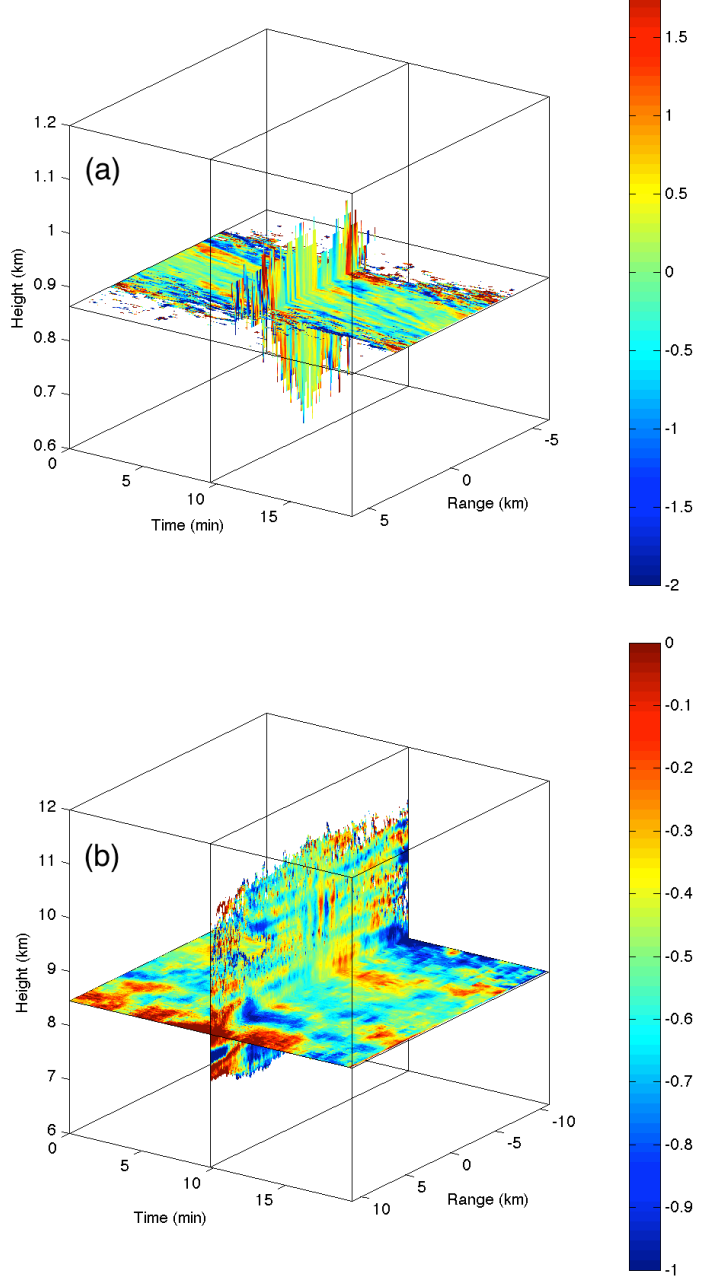

Fig. 13. 3-D representation of the retrieved vertical Doppler velocities from a set of CW-RHI scans for (a) a stratocumulus case and (b) a cirrus case. The horizontal cross section is near the middle of the cloud layer and the vertical section half way through the set.

range and (ii) identify a radar reflectivity threshold $\mathrm{dBZ}_{\text {thres }}$ (i.e., before we reach the radar sensitivity regime where $\partial \mathrm{CF}_{\mathrm{Hor}} / \partial \mathrm{dBZ}$ is large) that is sufficient to observe the bulk of the cloud structure of interest. Since the selection of a radar reflectivity threshold determines also the maximum range in which this sensitivity is achievable for the KaSACR, this approach could lead to a variable domain of 3-D gridded products. Different products could also lead to different 3-D gridded domains. For example, if we want to map 3-D drizzle structures, a large gridding domain is possible.

The suitability of a CW-RHI to address the need to describe the 3-D cloudy atmosphere is evaluated here. CWRHI observations from the Ka-band Scanning ARM Cloud Radar (SACR) are gridded in 3-D, with time being one of the dimensions. A method to retrieve the best estimate of the Ka-SACR vertical Doppler velocity component from the radial Doppler velocities is also presented. The Ka-SACR observations are initially post-processed in their native polarcoordinate system to (i) detect hydrometeors, (ii) correct for gaseous attenuation, (iii) remove Doppler velocity folding, and (iv) filter out insect echoes in the boundary layer. Subsequently, all CW-RHI polar-coordinate radar observations (all horizon-to-horizon scans) are gridded to a 2-D Cartesian grid. The gridding algorithm accounts for the 2-D beam geometry and identifies for each grid point all radar observations within its neighborhood. Then, all 2-D gridded radar observations are gridded along the time dimension. The grid domain size and the grid spacing are user-dependent. Four different gridding methods are available: maximum value, mean value, Cressman and Barnes. The Cressman and Barnes schemes produce very similar results. The possibility of a hybrid scheme that uses Cressman or Barnes everywhere but near the cloud edges is considered. Near cloud edges, the maximum value interpolation method performs best since the quality of the radar observables strongly depends on signalto-noise conditions.

Another important aspect that is evaluated here is the ability of the current CW-RHI scan strategy to resolve the 3-D morphological (i.e., as depicted by the radar reflectivity) and dynamical (i.e., as depicted by the Doppler velocity) structure of layer clouds. Comparisons of the time-height composite generated by extracting the vertically pointing beams of the Ka-SACR with the time-height composites from the dedicated profiling cloud radars at the ARM sites clearly indicates that although we are scanning from horizon to horizon with a relatively slow antenna scan rate ( 9 degrees per second), the Ka-SACR is able to resolve the largest eddies in the boundary layer cloud that are responsible for most of the turbulent transport of momentum, heat and mass.

Gridded radar reflectivity fields can be used to study 3D cloud inhomogeneity. However, off-nadir radar Doppler measurements critically depend on the projection of the horizontal wind to the radar line of sight. Thus, the observed KaSACR Doppler velocities are decomposed into the contribution of the horizontal wind and the contribution of the vertical Doppler velocity projected to the radar line of sight. The decomposition technique is applied to the 3-D gridded Doppler range-height velocity fields independently. At each individual height, the Doppler velocity measurements at angles $30^{\circ}$ to $75^{\circ}$ and $105^{\circ}$ to $150^{\circ}$ are used to estimate $V_{\mathrm{H}, \mathrm{RHI}}$ and its gradient (if any) along the scan plane, while the Doppler measurements at elevation angles $75^{\circ}$ to $105^{\circ}$ are used to estimate the mean Doppler velocity offset due to particle sedimentation. Finally, the contribution of the horizontal wind is removed and the residual Doppler velocities are corrected for radar centricity (divided with the sine of the elevation angle) to retrieve a best estimate of the vertical Doppler velocity component. The CW-RHI scan strategy can only focus on a particular cloud layer/type since typically the wind direction changes with height. Thus, a prioritization based on the scientific objectives tied to the operation of the SACR 
at the ARM site is needed to select a particular cloud type (e.g., boundary layer clouds, cirrus clouds) or atmospheric layer (e.g., middle or upper troposphere) that will be of interest. This information along with the profile of the horizontal wind will be available in the data set to help potential data users

Analysis of the standard deviation of the retrieved 3-D $V_{\mathrm{D}, \mathrm{V}}$ field suggests that the retrievals of $V_{\mathrm{D}, \mathrm{V}}$ are noisy for elevation angles lower than $60^{\circ}$ or higher than $120^{\circ}$. Despite this limitation, the best estimate of the vertical Doppler velocity component provides unique information on the 3-D coherent dynamical structures in clouds. Such information cannot be obtained from profiling cloud radars and can lead to an improved understanding of cloud dynamics and their relationship to large-scale meteorology; however, one must consider that the gridding of the radar observables (Doppler velocity and radar reflectivity) is recommended for the purpose of investigating the clouds' 3-D morphology and dynamical structure. Microphysical retrievals, especially those based on dual-wavelength measurements available from the SACRs, should be performed at the SACR native coordinate system since small changes in the SACR observables due to the gridding can have a large effect on retrievals.

Acknowledgements. This research was funded by the Office of Biological and Environmental Research, Environmental Sciences Division of the US Department of Energy, as part of the Atmospheric Systems Research (ASR) program. We would also like to thank the entire McGill Clouds group for their feedback and support.

Edited by: A. Kokhanovsky

\section{References}

Askelson, M. A.: A Study of the Kinematic and Microphysical Evolution of a Supercell from First Echo using Polarization Diversity Radar, School of Meteorology, 94. 100 E. Boyd, Norman, Ok 73019, University of Oklahoma, 1996.

Barnes, S. L.: A technique for maximizing details in numerical weather map analysis, J. Appl. Meteorol., 3, 396-409, 1964.

Browning, K. and Wexler, R.: The determination of kinematic properties of a wind field using Doppler radar, J. Appl. Meteorol., 7, 105-113, 1968.

Clothiaux, E., Miller, M., Albrecht, B., Ackerman, T., Verlinde, J., Babb, D., Peters, R., and Syrett, W.: An evaluation of a 94-GHz radar for remote sensing of cloud properties, J. Atmos. Ocean. Tech., 12, 201-229, 1995.

Clothiaux, E. E., Ackerman, T. P., Mace, G. G., Moran, K. P., Marchand, R. T., Miller, M. A., and Martner, B. E.: Objective determination of cloud heights and radar reflectivities using a combination of active remote sensors at the ARM CART sites, J. Appl. Meteorol., 39, 645-665, 2000.

Cressman, G. P.: An operational objective analysis system, Mon. Weather Rev., 87, 367-374, 1959.
Davis, A., Marshak, A., Cahalan, R., and Wiscombe, W.: The Landsat scale break in stratocumulus as a three-dimensional radiative transfer effect: Implications for cloud remote sensing, J. Atmos. Sci., 54, 241-260, 1997.

Deng, M. and Mace, G. G.: Cirrus microphysical properties and air motion statistics using cloud radar Doppler moments. Part I: Algorithm description, J. Appl. Meteorol. Clim., 45, 1690-1709, 2006.

Doviak, R. J. and Zrnić, D. S.: Doppler Radar and Weather Observations, Academic Press, 1993.

Frisch, A., Fairall, C., and Snider, J.: Measurement of stratus cloud and drizzle parameters in ASTEX with a $K \alpha$-band Doppler radar and a microwave radiometer, J. Atmos. Sci., 52, 2788-2799, 1995.

Fulton, R. A.: WSR-88D polar-to-HRAP mapping, National Weather Service, Hydrologic Research Laboratory, Technical Memorandum, 9-9, 1998.

Hildebrand, P. H. and Sekhon, R. S.: Objective Determination of Noise Level in Doppler Spectra, J. Appl. Meteorol., 13, 808-811, 1974.

Hinkelman, L. M., Stevens, B., and Evans, K. F.: A large-eddy simulation study of anisotropy in fair-weather cumulus cloud fields, J. Atmos. Sci., 62, 2155-2171, 2005.

Jorgensen, D. P., Hildebrand, P. H., and Frush, C. L.: Feasibility test of an airborne pulse-Doppler meteorological radar, J. Clim. Appl. Meteorol., 22, 744-757, 1983.

Kollias, P. and Albrecht, B.: The turbulence structure in a continental stratocumulus cloud from millimeter-wavelength radar observations, J. Atmos. Sci., 57, 2417-2434, 2000.

Kollias, P., Clothiaux, E., Miller, M., Albrecht, B., Stephens, G., and Ackerman, T.: Millimeter-wavelength radars: New frontier in atmospheric cloud and precipitation research, B. Am. Meteorol Soc., 88, 1608-1624, 2007.

Kollias, P., Bharadwaj, N., Widener, K., Jo, I., and Johnson, K.: Scanning ARM Cloud Radars (SACR's) Part I: Operational Sampling Strategies, J. Atmos. Ocean. Tech., doi:10.1175/JTECH-D13-00044.1, in press, 2013a.

Kollias, P., Jo, I., Borque, P., Tatarevic, A., Lamer, K., Bharadwaj, N., Widener, K., Johnson, K., and Clothiaux, E.: Scanning ARM Cloud Radars - Part II: Data Quality Control and Processing, J. Atmos. Ocean. Tech., doi:10.1175/JTECH- D-13-00045.1, in press, 2013b.

Lhermitte, R. M. and Atlas, D.: Precipitation motion by pulse Doppler radar, Proc. Ninth Weather Radar Conf, 218-223, Am. Meteorol. Soc. Boston, MA, 1961.

Mather, J. H. and Voyles, J. W.: The ARM climate research facility: A review of structure and capabilities, B. Am. Meteorol. Soc., 94, 377-392, 2013.

Miller, J. L., Mohr, C. G., and Weinheimer, A. J.: The simple rectification to Cartesian space of folded radial velocities from Doppler radar sampling, J. Atmos. Ocean. Technol., 3, 162-174, 1986.

Mohr, C. and Vaughan, R.: An economical procedure for Cartesian interpolation and display of reflectivity factor data in threedimensional space, J. Appl. Meteorol., 18, 661-670, 1979.

Nelson, S. P.: A study of hail production in a supercell storm using a Doppler derived wind field and a numerical hail growth model, Ph.D. thesis, School of Meteorology, Vol. 41-05, Section: B, p. 1806, 1980. 
Qian, Y., Long, C. N., Wang, H., Comstock, J. M., McFarlane, S. A., and Xie, S.: Evaluation of cloud fraction and its radiative effect simulated by IPCC AR4 global models against ARM surface observations, Atmos. Chem. Phys., 12, 1785-1810, doi:10.5194/acp-12-1785-2012, 2012.

Randall, D. A., Xu, K.-M., Somerville, R. J., and Iacobellis, S.: Single-column models and cloud ensemble models as links between observations and climate models, J. Climate, 9, 16831697, 1996.

Rémillard, J., Kollias, P., Luke, E., and Wood, R.: Marine Boundary Layer Cloud Observations in the Azores, J. Climate, 25, 73817398, 2012.

Rosenkranz, P. W.: Water vapor microwave continuum absorption: A comparison of measurements and models, Radio Sci., 33, 919928, 1998.

Shupe, M. D., Walden, V. P., Eloranta, E., Uttal, T., Campbell, J. R., Starkweather, S. M., and Shiobara, M.: Clouds at Arctic atmospheric observatories. Part I: Occurrence and macrophysical properties, J. Appl. Meteorol. Clim., 50, 626-644, 2011.
Somerville, R. and Iacobellis, S.: Single-column models, ARM observations, and GCM cloud-radiation schemes, Phys. Chem. B, 24, 733-740, 1999.

Stokes, G. M. and Schwartz, S. E.: The Atmospheric Radiation Measurement (ARM) Program: Programmatic background and design of the cloud and radiation test bed, B. Am. Meteorol. Soc., 75, 1201-1221, 1994.

Trapp, R. J. and Doswell III, C. A.: Radar data objective analysis, J. Atmos. Ocean. Tech., 17, 105-120, 2000.

Zhang, J., Howard, K., and Gourley, J.: Constructing threedimensional multiple-radar reflectivity mosaics: Examples of convective storms and stratiform rain echoes, J. Atmos. Ocean. Tech., 22, 30-42, 2005.

Zinner, T. and Mayer, B.: Remote sensing of stratocumulus clouds: Uncertainties and biases due to inhomogeneity, J. Geophys. Res., 111, D14209, doi:10.1029/2005JD006955, 2006. 


\section{Appendix A}

\section{Interpolation schemes available for gridding Ka-SACR data}

\section{A1 Maximum value scheme}

The value in the grid point is determined by the observational value having a maximal reflectivity among the observational values "influencing" the value at this grid point. Let $N_{k}$ be the total number of observational points $F^{\text {obs }}$ having an influence on the value $F_{\mathrm{g}}$ at grid point $x_{i x}, z_{i z}$, and let $d_{(i, j), k}$ be the distance between the grid point $F_{\mathrm{g}}$ and $k$ th observational point $\left(x_{i, j}, z_{i, j}\right){ }_{k}$, where $k \in\left(1, N_{k}\right)$. The value $F_{\mathrm{g}}$ at the grid point $x_{i x}, z_{i z}$ is given by

$$
F_{\mathrm{g}}\left(x_{i x}, z_{i z}\right)=\operatorname{Max}\left\langle F_{(i, j), k}^{\mathrm{obs}}\right\rangle .
$$

\section{A2 Mean value scheme}

The value in the grid point is determined by averaging the influencing observational values:

$F_{\mathrm{g}}\left(x_{i x}, z_{i z}\right)=\frac{\sum_{k}^{N_{k}} F_{(i, j), k}^{\mathrm{obs}}}{N_{k}}$.

\section{A3 Cressman-type method}

The value $F_{\mathrm{g}}$ at the grid point $x_{i x}, z_{i z}$ is given by

$F_{\mathrm{g}}\left(x_{i x}, z_{i z}\right)=\frac{\sum_{k}^{N_{k}} w_{(i, j), k}^{(i x, i z)} F_{(i, j), k}^{\mathrm{obs}}}{\sum_{k}^{N_{k}} w_{(i, j), k}^{(i x, i z)}}$,

with the Cressman weighting function $w$ at the grid point $\left(x_{i x}, z_{i z}\right)$ given as

$w_{(i, j), k}^{(i x, i z)}=\frac{R_{(i, j), k}^{2}-d_{(i, j),(i x, i z), k}^{2}}{R_{(i, j), k}^{2}+d_{(i, j),(i x, i z), k}^{2}}, \quad$ for $\quad d^{2} \leq R^{2}$,

$w_{(i, j), k}^{(i x, i z)}=0$, for $d^{2}>R^{2}$,

where $d^{2}$ is the distance from the center of radar resolution volume $r_{i}, \theta_{j}$ of the $k$ th observational point, $k \in\left(1, N_{k}\right)$, to the grid point $x_{i x}, z_{i z}$. The weight of a measurement is equal to 1 if it is located exactly on the grid point and equal to 0 on the edge of and outside the sphere with radius $R$.

The radius of influence $R_{(i, j), k}$ depends on the size of the radar resolution volume relative to the grid resolution, and is defined as

$R_{(i, j), k}=\operatorname{Max}\left[R_{d 1}, R_{d 2}\right]$, where

$$
\begin{aligned}
& R_{d 1}=\left[\frac{\Delta x^{2}+\Delta z^{2}}{4}\right]^{0.5}, \\
& R_{d 2}= \\
& \quad\left[\mathrm{dr}^{2}+\left(r_{i}+0.5 \mathrm{dr}\right)^{2} \sin ^{2}\left(0.5 \operatorname{Max}\left\{\theta_{j+1}-\theta_{j}, b w\right\}\right)\right]^{0.5} .
\end{aligned}
$$

In the standard approach, for each grid point all observational values within a specified radius of influence are used as an input to a gridding scheme and its associated weighting function (e.g., Barnes, Cressman, etc.). While large values of the radius of influence increase the smoothing effects, very small values can create gaps in gridded data. Large values of the radius of influence can also introduce artificial values at grid points corresponding to observational cloud-free pixels, especially near cloud boundaries. With these choices regarding the radius of influence, the effect of smoothing in gridded data primarily depends on the choice of grid resolution. Note that only cloud pixels are examined when selecting the observational values that "influence" the value at a specific grid point. An implicit consequence of the selection method is that the discontinuity in observed data, if larger than the grid resolution, is preserved. When lowering the grid resolution in $x$ and $z$, the smoothing takes effect and cloud boundaries tend to spread out. Although grid spacing is a parameter defined by the user, the application of high grid resolution in gridding algorithms is implicitly stipulated and advised.

\section{A4 Barnes-type method}

The method is similar to Cressman, except a different weighting function is used:

$\begin{array}{ll}w_{(i, j), k}^{(i x, i z)}=\exp \left(-\frac{d_{(i, j),(i x, i z), k}^{2}}{2 R_{(i, j), k}^{2}}\right), & \text { for } \quad d^{2} \leq R^{2}, \\ w_{(i, j), k}^{(i x, i z)}=0, & \text { for } \quad d^{2}>R^{2} .\end{array}$

The weighting function (A7) is defined as in Trapp and Doswell III (2000), with the smoothing parameter defined as two times the characteristic data spacing squared. The method is expected to produce similar results to those produced by the Cressman-type method. 